\title{
De la enseñanza con libros de texto al aprendizaje en espacios online gamificados
}

\section{From Teaching with Textbooks to Learning on Online Gamified Spaces}

\author{
Manuel Area Moreira \\ manarea@ull.edu.es \\ Carina S. González González \\ cjgonza@ull.edu.es \\ Universidad de La Laguna, España
}

\section{Resumen:}

En este artículo se plantea la necesidad que la escuela del siglo XXI empiece a transformar sus materiales didácticos adecuándolos a las nuevas experiencias y formas expresivas de la sociedad digital. Comienza con el análisis del medio o material didáctico idiosincrático escolar como es el libro de texto. Se le caracteriza como un producto derivado de una concepción enciclopedista del curriculum, derivado del modelo de las industrias culturales del siglo XX y que juega un papel mediador entre el curriculum oficial y la práctica docente ya que vertebra lo que debe enseñarse y aprenderse. Frente a ello, presentamos un nuevo enfoque de producción de materiales educativos basados en la lógica de los videojuegos. Es el enfoque de gamificación del aprendizaje que implica un modelo de aprendizaje basado en situaciones problemáticas, en mayor interactividad humano-máquina y con componentes lúdicos. Concluimos que estos materiales digitales gamificados tienen profundas implicaciones para la reestructuración de las industrias editoras, así como para las concepciones y prácticas de enseñanza y aprendizaje en las escuelas.

\begin{abstract}
:
This article raises the need for twenty-first century schools to begin transforming their teaching materials by adapting them to the new experiences and expressive forms offered by the digital society. Our study begins by analysing an idiosyncratic school means--textbooks. Text-books are underlying by an encyclopedic understanding of the curriculum made popular by twentieth century's cultural industries and they play an important mediating role between the official curriculum and teaching practice since they scaffold what should be taught and learned. As an alternative to text-books, we present a new approach to producing educational materials based on the logic of video games: gamification. This approach to learning involves a model based on problem solving, on a better human-machine interactivity and on ludic components. Our conclusion is that these digital gamified materials have profound implications for the restructuring of the publishing industry, as well as for the conceptions and practices of teaching and learning in schools.
\end{abstract}


Palabras clave:

Material didáctico; libro de texto; videojuegos; gamificación; TIC.

\section{Keywords:}

Teaching materials; textbooks; video games; gamification; ICT.

\section{Résumé:}

Cet article soulève la nécessité que l'école du XXIe siècle commence à transformer leurs matériels d'enseignement en les adaptant aux nouvelles expériences et aux formes d'expression de la société numérique. Il commence par analyser le milieu ou le matériel didactique idiosyncrasique, c'est-à-dire, le manuel scolaire. Il se caractérise comme un sous-produit de la conception encyclopédique du"curriculum", qui est un modèle dérivé des industries culturelles du XXe siècle et qui a joué un rôle médiateur entre le programme officiel et et pratique de l'enseignement étant donné qu'il vertèbre ce qui doit être enseigné et appris. Face à cela, nous présentons une nouvelle approche de la production de matériel pédagogique basé sur la logique des jeux vidéo. C'est liapproche de la gamification de lıapprentissage qui implique un modèle d`apprentissage basé sur des situations problématiques, avec plus dsinteraction homme-machine et des composants ludiques. Nous concluons que ces matériels numériques ont des implications profondes pour la restructuration de lindustrie de liédition, ainsi que pour les conceptions et les pratiques dienseignement et diapprentissage dans les écoles.

\section{Mots-clés:}

Matériel didactique; les manuels; les jeux vidéo; la gamification; TIC.

Fecha de recepción: 6-1-2015

Fecha de aceptación: 3-7-2015

\section{Introducción: Del libro de texto como material didáctico idiosincrático de la escuela del $\mathrm{XX}$ a los entornos digitales de la escuela del XXI}

El libro de texto de papel puede ser considerado como el invento tecnológico más genuino e idiosincrático de la institución escolar nacida a mediados del siglo XIX. El libro de texto, además de ser un artefacto o recurso pudiéramos etiquetarlo como la tecnología educativa apropiada para un modelo de escolaridad basada en la transmisión expositiva del conocimiento, en el aprendizaje individual, en un curriculum segmentado por asignaturas y organizado en función de edades y niveles (Martínez Bonafé, 2001, 2008, 2010).

La escuela y el libro de texto, a lo largo de estos dos últimos siglos, han constituido una simbiosis tan intensa que hoy en día resulta muy difícil de romper. El hábitat natural de texto escolar es un aula con un docente que explica y unos alumnos que aprenden a partir de libros. En 
otro modelo de práctica escolar el libro de texto no tiene sentido y funcionalidad. De modo similar, ocurre con la escuela tradicional ya que para funcionar necesita de materiales didácticos, en formato libro individual para cada estudiante, que establezcan y ofrezcan secuenciadamente a lo largo de todo el curso académico los contenidos y las actividades que deben cumplimentarse en el ámbito del aula.

A lo largo del siglo XX estas reglas de juego estuvieron claramente definidas, eran funcionales ya que permitían lograr, en mayor o menor medida, las metas educativas establecidas en el curriculum oficial y sobre todo estaban asumidas, sin que fueran cuestionadas, por todos los agentes educativos: los docentes, los alumnos, las familias, las administraciones gubernamentales, las empresas productoras de materiales, los políticos y toda la sociedad en general.

Sin embargo, la irrupción y expansión de la tecnología digital, en sus múltiples formatos (ordenadores personales, tablets, telefonía móvil, gadgets, ...) está transformando sustantivamente no sólo los modos tradicionales de producción, almacenamiento, difusión y consumo de información, sino que también empieza a entrar en las escuelas alterando el estatus quo de los modelos tradicionales de enseñanza y de su material didáctico.

Las políticas educativas destinadas a la introducción de las TIC en las escuelas desarrolladas desde hace más de dos décadas en Europa y, en particular, la implantación del modelo 1:1 (Balanskat, Bannister, Hertz, Sigillò \& Vuorikari, 2013), es decir, de una dispositivo tecnológico por estudiante, están propiciando que empiecen a ser habitual en las aulas el desarrollo de experiencias de aprendizaje basadas en el uso de las tecnologías digitales. El ya desaparecido Programa Escuela 2.0 facilitó en el contexto español un incremento de la tecnología disponible en las aulas (Area y Sanabria, 2014).

En este artículo pretendemos analizar estos profundos procesos de transformación de un material didáctico tradicional como es el libro de texto describiendo sus características y funcionalidades en el curriculum hacia un nuevo enfoque y concepción del material educativo basado en interfaces digitales e interactivas que plantean situaciones lúdicas y problemáticas similares a la lógica de funcionamiento de los videojuegos. 


\section{La funcionalidad del libro de texto como tecnología educativa de la escuela enciclopedista e industrial}

El estatus de privilegio y monopolio del libro de texto en la vida académica de las escuelas podría explicarse por la conjunción de distintos factores o fenómenos que van más allá de las características técnicas de estas obras impresas y que son producto de fenómenos de naturaleza sociocultural y pedagógica. La funcionalidad y éxito del libro de texto como tecnología educativa, en el modelo de escolaridad existente hasta la fecha viene dado, entre otras razones, por lo que se presenta en la figura 1.

\section{a) El libro de texto como tecnología apropiada}

El libro de texto es una tecnología apropiada para un modelo de enseñanza expositiva del conocimiento y de aprendizaje por recepción. El concepto de tecnología apropiada viene ya de varias décadas atrás (Klassen y Solid, 1981) y hace referencia a que ciertas tecnologías se adecuan o funcionan eficazmente en determinados contextos o situaciones. En nuestro caso, el texto escolar es la tecnología apropiada al modelo de escolaridad tradicional donde la visión o teoría pedagógica dominante es la de enseñar a través de métodos expositivos y de aprendizaje por recepción. Este material didáctico es la tecnología adecuada para ello ya que ofrece una selección del conocimiento disponible para una determinada materia o asignatura, el mismo está organizado y presentado de forma secuencial y adaptado, en mayor o menor medida, tanto en su vocabulario y en su grado de dificultad a sus potenciales usuarios.

Dicho de otro modo, el libro de texto es el eje vertebrador o piedra angular del método expositivo en la educación escolar. Esta teoría pedagógica o concepción de enseñanza-aprendizaje impregnó la génesis de la institución escolar a mediados del siglo XIX y se ha consolidado en el $\mathrm{XX}$ de forma que tanto los docentes como los estudiantes nunca la han cuestionado.

Sin embargo, desde hace más de un siglo, los expertos y la investigación procedente tanto de la psicología como pedagogía establecieron otras teorías y enfoques educativos (sobre todos los autores de la denominada Escuela Nueva y Moderna como Decroly, Dewey, Piaget, Freinet, ...) que chocaban o contradecían la teoría hegemónica de las 
prácticas escolares basadas en la transmisión de información bien vía oral -a través de exposiciones del docente al conjunto de la clase-, bien vía materiales como el libro de texto. En este sentido, como veremos más adelante, la irrupción de las tecnologías digitales y de los entornos online abren nuevas oportunidades para que las teorías pedagógicas de la Escuela Nueva y de la psicología constructivista se generalice y sea una práctica habitual en las escuelas.

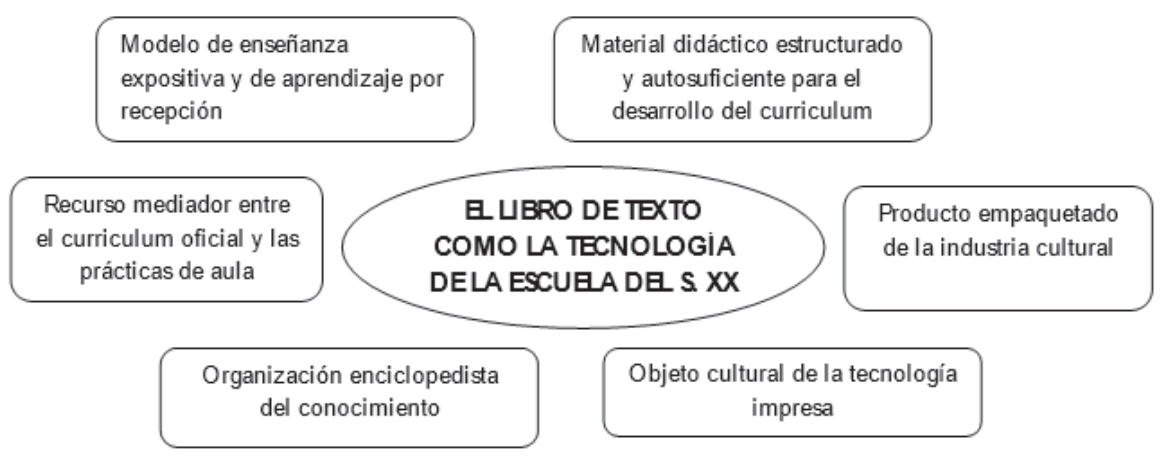

Figura 1

\section{b) El libro de texto como material curricular estructurado que prescribe la práctica escolar}

El curriculum escolar, en la mayor parte de los países, ha sido concebido y elaborado como un instrumento de prescripción y control, por parte de las autoridades educativas, de las prácticas educativas de las aulas. Los trabajos de la denominada sociología crítica del curriculum han puesto de manifiesto que los poderes gubernamentales han utilizado al curriculum como el mecanismo que regula y estructura lo que se enseña y aprende en las escuelas.

La gramática de la mayor parte de los curricula oficiales adopta un formato de presentación de los objetivos y contenidos que guíe o prescriba la práctica del profesorado. En esta lógica, el libro de texto se convirtió en el recurso o material más útil para trasladar a las aulas las directrices curriculares. Gimeno (1998) denominó a este fenómeno como la función traductora entre el curriculum oficial y el curriculum en acción. El libro de texto operativiza en términos concretos de práctica diaria de trabajo en el aula lo que son principios y supuestos educativos estable- 
cidos en los documentos oficiales y formulados en términos genéricos. El texto escolar es el único material didáctico que está concebido y elaborado para estructurar y dar forma a un proyecto de trabajo docente para un curso escolar completo. Es una propuesta didáctica cerrada y autosuficiente de modo que el papel del profesor consiste en aplicarla a su grupo de estudiantes.

\section{c) El libro de texto como producto de la industria cultural educativa}

El nacimiento de la escuela como institución social regulada por el estado moderno es coetánea con la aparición y desarrollo de la industrialización. El oficio de enseñar, en consecuencia, pasó de ser una actividad artesanal y desregulada a ser una tarea ofertada por el estado con la finalidad de formar en masa a la mayor cantidad de población infantil y juvenil de un determinado país. Por ello, la estandarización de la oferta formativa, el establecimiento de criterios de homogeneidad en la selección y promoción de los estudiantes, así como garantizar que todos los escolares recibieran los mismos contenidos, propició que hubiera un único material que garantizaba que todos los docentes, todos los alumnos y todas las aulas del país siguieran el mismo proceso y enseñaran los mismos conocimientos. Este material que permitía uniformar estos fenómenos fue el texto escolar.

Su lógica de producción, distribución y consumo fue asumida por industrias o empresas privadas especializadas en este tipo de productos culturales creados ex profeso para la actividad escolar. Hasta la fecha este mercado de los libros de texto y otros materiales didácticos ha funcionado sin grandes sobresaltos, pero los tiempos están cambiando y esta industria cultural, al igual que otras similares vinculadas con el ocio, la música, la literatura, el cine o la prensa están en crisis por la explosión y omnipresencia de las tecnologías digitales.

Estas han roto las reglas de juego tradicionales con relación a quiénes producen información, cómo se difunde y cómo se accede al consumo de la misma. Frente al modelo industrial donde los productores eran empresas privadas y donde los usuarios para poder consumir cualquier producto cultural (un libro, un periódico, una película, un disco) tenían que comprarlo, la tecnología digital está propiciando que cualquier sujeto puede convertirse fácilmente en productor de información y difundirla en la red, así como la posibilidad de reproducir la misma obra digital- 
mente lleva a que ésta sea fácilmente distribuida y accesible provocando que, en muchas ocasiones, su consumo no requiera pagar por la misma. Esto nos lleva al concepto creciente de licencias copyleft y de recursos libres y de acceso abierto, lo que tiene unas enormes repercusiones sobre la naturaleza y potencialidades de los nuevos materiales didácticos distribuidos digitalmente. Este sentido, el libro de texto como objeto o mercancía cultural, deja de tener sentido para abrir un nuevo tipo de materiales educativos online generados por los propios docentes basados en el intercambio, en el acceso libre y en su reutilización o recreación sin restricciones comerciales.

\section{d) El libro de texto como objeto de la cultura impresa}

El libro de texto es, ante todo, un libro impreso, un objeto tecnológico de papel. La institución escolar, desde su creación a mediados del siglo XIX, siempre tuvo al libro impreso como canon cultural, como referente fundamental para su función educativa. La escuela moderna está construida alrededor del libro como objeto cultural. La escolaridad siempre fue, y sigue siendo, sinónimo de alfabetización en la lectoescritura ya que la misma es la competencia indispensable para continuar en el sistema formativo y adquirir la condición de persona culta. Precisamente esta meta, la erradicación del analfabetismo, es la que legitimó la utilidad social de la escuela ya que la alfabetización es una condición necesaria para el progreso social y la democratización de la cultura. El texto escolar, en consecuencia, se convirtió en ese libro específico e idiosincrático que sintetizaba, organizaba y mostraba el saber que debía ser enseñado y aprendido en las aulas.

La institución escolar es una construcción de la sociedad moderna porque, entre otras razones, adoptó y se apropió de la tecnología del papel sobre la que se edificó la racionalidad y el pensamiento de la modernidad occidental. Escuela y tecnología impresa se convirtieron en un binomio inseparable hasta la fecha. Las escuelas por una parte, junto a las bibliotecas por otra, fueron los espacios públicos de culto al libro como objeto cultural. El papel de la escuela era formar a los lectores, la función de la biblioteca era custodiar los libros y facilitar el acceso democrático a los mismos.

Durante muchas décadas se consideró que el curriculum escolar debía encargarse de transmitir el conocimiento cultural que estaba en los 
libros, desconsiderando otro tipo de manifestaciones culturales, muy relevantes en la segunda mitad del siglo XX, en formatos diferentes del papel como eran la música en discos, los audiovisuales, la cinematografía y la fotografía entre otros. Hoy en día, es evidente, que la escuela no puede seguir dando la espalda a las nuevas tecnologías digitales ya que éstas, entre otras razones, han fagocitado a todos los demás medios hasta hacerlos prácticamente desaparecer. La cultura, hoy en día, es digital.

\section{e) El libro de texto como enciclopedia}

Paralelamente a la expansión en Europa de la tecnología impresa y a las ideas que insertas en las páginas de los libros, a finales del siglo XVIII, empezó a gestarse una nueva visión del saber basada más en la razón que en la fe, apoyada más en las evidencias y certezas empíricas que en las verdades reveladas. El movimiento francés de esa época conocido como llustración estableció la arquitectura del pensamiento racional de Occidente tal como ha llegado hasta nuestro presente. Uno de los objetivos del movimiento ilustrado fue recopilar, dar forma y organizar de modo sistemático lo que era el conocimiento humano. Este proceso consistió en escribir la "Enciclopedia" (1751-1772) es decir, un conjunto de libros que pretendían clasificar y organizar el saber humano para hacerlo accesible al público. El eje clave de esta organización del saber fueron las materias o campos científicos.

Este modelo organizativo fue el que inspiró y dio forma al curriculum escolar un siglo después. Éste, en consecuencia, fue dividido en partes (las materias y asignaturas) estableciéndose los contenidos que debían ser enseñados. En esta lógica, el libro de texto apareció como una "pequeña enciclopedia" adaptada a las necesidades formativas del alumnado escolar en función de su nivel de conocimientos y edad.

El libro de texto es hijo o consecuencia de una concepción ilustrada y enciclopedista en la organización del curriculum donde ha primado más el conocimiento disciplinar que las competencias o ámbitos de aprendizaje de los sujetos. Esta concepción disciplinar del curriculum -vigente hasta nuestros días- requiere, como acabamos de señalar, para su puesta en práctica en las aulas de "minienciclopedias" especificas para cada materia y nivel educativo. 


\section{f) Los libros de texto y la profesionalidad docente}

Finalmente un último fenómeno que explica la hegemonía del texto escolar en las prácticas de aula está vinculado con su función mediadora curricular que nombramos anteriormente y el papel del profesorado en la misma. Nos referimos a que los libros de texto encierran una determinada teoría o visión de la profesionalidad docente (Martinez Bonafé, 2010) que separa a los productores de los consumidores de estos materiales. La existencia y estatus quo de los libros de texto consolidan un modelo de descualificación profesional de los docentes basado en la división social del trabajo entre expertos o técnicos curriculares y maestros. Éstos, a través de los libros de texto, se convierten en meros aplicadores o gestores en su aula de propuestas de trabajo docente elaboradas en instancias ajenas y alejadas de su escuela. Los denominados materiales curriculares "a prueba de profesores" (como es el caso del texto escolar) son aquellos que son elaborados por expertos 0 empresas para ofrecer a los profesores productos o materiales que presentar una propuesta o proyecto educativo "empaquetado" con sus objetivos, contenidos, actividades y evaluación detallados.

De este modo, los libros de texto sustituyen al profesorado en el proceso de toma de decisiones de adecuación y operativización del curriculum a su grupo de estudiantes concretos. La labor del docente queda reducida a gestionar el uso que realizan los alumnos de los libros de texto en el aula y el hogar.

Por otra parte la carencia de la formación y competencias adecuadas, junto con las características limitantes del puesto de trabajo escolar, provoca que muchos docentes tengan dificultades para planificar, desarroIlar y evaluar proyectos educativos que vayan más allá de la mera transmisión de información. Intentar crear materiales propios alternativos a los libros de texto requiere de otro modelo de profesionalidad docente que implica mayores competencias para hacer frente a situaciones de mayor complejidad pedagógica.

\section{Cerrando los libros y abriendo los juegos: La gamificación del aprendizaje}

Actualmente, de forma muy rápida y en constante crecimiento, los videojuegos se están convirtiendo en una de las formas más populares de 
entretenimiento para todas las edades y géneros. Muchos videojuegos requieren que los jugadores aprendan y adquieran habilidades complejas, por ello implican el desarrollo de habilidades y procesos cognitivos superiores, siendo la motivación y el compromiso dos pre-requisitos necesarios para el logro de las tareas en el juego (Gee, 2003). Asimismo, la investigación constata que los videojuegos poseen numerosos efectos positivos, entre los que encontramos los de tipo instructivo (McFarlane, Sparrowhawk \& Heald, 2002). Por ello, creemos que la incorporación de elementos y mecánicas de los juegos en la educación podría disminuir los abandonos, la falta de motivación, el desgano y la falta de compromiso con el proceso de enseñanza, al mismo tiempo que se potenciaría el aprendizaje de competencias (Mérida, Angulo, Jurado \& Díaz, 2011). A este proceso lo denominamos "gamificación" aplicada a la Educación, y con ella se busca promover la motivación, el compromiso y ciertos comportamientos en las personas (Lee \& Hammer, 2011).

En la actualidad no existe un consenso general sobre la definición del término "gamificación", sin embargo la idea principal de utilizar elementos del diseño de juegos en entornos no lúdicos con el fin de que las personas puedan adquirir conocimientos y destrezas propias de su entorno de manera divertida, está ganando cada vez más adeptos y según Gartner (2012) es una de las tecnologías emergentes.

Existen trabajos relacionados con la gamificación en la educación que indican algunos beneficios de introducir estas mecánicas en las aulas (González \& Blanco, 2008; Fitz-Walter, Tjondronegoro \& Wyeth, 2011; Barata, Gama, Jorge \& Gonçalves, 2013; Zhijiang, Untch \& Chasteen, 2013; González \& Mora, 2014). Así, González y Blanco (2008) proponen aumentar la motivación y colaboración en los entornos de elearning, tales como Moodle combinando estrategias de videojuegos como las de juegos de rol online. Fit-Walter y otros (2011) presentan una experiencia de m-learning realizada para estudiantes recién ingresados en la universidad en donde aplican un modelo de diseño de logros de forma satisfactoria. En Barata y otros (2013) se describe un experimento en un curso de ingeniería, en donde se utilizan 6 elementos de gamificación: puntos de experiencia, niveles, tablas de clasificación, desafíos, insignias, y árbol de habilidades. Zhijiang y otros (2013) utilizan diferentes elementos de gamificación para aumentar la participación de los estudiantes de informática en una red social, como ambiente de aprendizaje colaborativo. A través de la gamificación se busca aumentar la interac- 
ción de los estudiantes sobre temas relacionados con el curso, así como otros temas puramente sociales. Por último, González y Mora (2014) presentan una propuesta educativa metodológica basada en la introducción de técnicas de gamificación o mecánicas de juego en el proceso de enseñanza-aprendizaje de Ingeniería Informática. Además, presentan una guía de técnicas y estrategias basadas en las mejores prácticas de gamificación en la educación. Estos autores presentan sus estrategias de gamificación como estrategias didácticas, y brindan pautas y guías para el diseño de actividades educativas gamificadas.

En este sentido, Merquis (2013) indica que la gamificación en la educación puede aportar a los estudiantes mejores oportunidades para desarrollar:

- Compromiso: básicamente, hace que los estudiantes estén más interesados en lo que están aprendiendo;

- Flexibilidad: la incorporación de elementos de gamificación permite a los estudiantes desarrollar una mayor flexibilidad mental y habilidades de resolución de problemas;

- Competición: los juegos y los elementos del aprendizaje basado en el juego, están íntimamente relacionados con el deseo natural del ser humano para la competencia, que en este caso, permite a los estudiantes aprender de sus errores y no ser penalizados por ellos;

- Colaboración: en un mundo hiperconectado, los estudiantes deben ser capaces tanto de colaborar con los demás compañeros de forma local, así como en línea.

Apoyándonos en las investigaciones anteriores, nos preguntamos entonces: ¿qué elementos de juego o mecánicas se pueden utilizar para mejorar la participación de los estudiantes de ingeniería en un modelo de enseñanza-aprendizaje b-learning o también denominada semi-presencial? y ¿la gamificación de las actividades educativas puede mejorar los resultados de los estudiantes en una asignatura?

Para dar respuesta a estas preguntas, primero veremos algunas de las mecánicas de videojuegos más utilizadas en la educación, y luego cómo podemos introducirlas en las actividades educativas que forman parte de la propuesta metodológica que aquí se presenta y se pretende desarrollar en el aula de clases, así como fuera de ella, siguiendo el modelo b-learning. 


\section{Fundamentos de la propuesta de gamificación en la educación}

Tal como vimos en el apartado anterior, esencialmente, la gamificación intenta aplicar la mecánica de los juegos en otros entornos, como el educativo. Aunque muy relacionados, el concepto de gamificación no está directamente relacionado con el diseño de videojuegos, sino con el componente adictivo de los videojuegos, que aplicado en entornos diferentes pretende atraer al usuario y lograr que realice ciertas acciones de forma satisfactoria. Podemos decir entonces que para gamificar una actividad hay que pensar como un diseñador de juegos.

Según Cook (2013), cualquier proceso que cumpla las siguientes premisas puede ser transformado en un juego o ser gamificado: a) la actividad puede ser aprendida; b) las acciones del usuario pueden ser medidas y c) los feedbaks pueden ser entregados de forma oportuna al usuario. Por tanto, vemos factible que las actividades formativas pueden ser gamificadas.

Básicamente, la gamificación intenta satisfacer algunos de los deseos o necesidades humanas fundamentales que la gente necesita, tanto en el mundo real como en el virtual, tales como: el reconocimiento, la recompensa, el logro, la competencia, la colaboración, la autoexpresión y el altruismo. Para ello, utiliza distintos elementos que junto a la estética del juego, crearán la experiencia del jugador o jugadora. Según Kevin Werbach (2012), los tres elementos son las dinámicas, las mecánicas y los componentes, y podemos verlos en una estructura piramidal, dependiendo de si el elemento es táctico o conceptual (Figura 1). Las dinámicas son el concepto, la estructura implícita del juego. Las mecánicas son los procesos que provocan el desarrollo del juego y pueden ser de distintos tipos, tales como:

a) mecánicas sobre el comportamiento (centrado en el comportamiento humano y la psique humana),

b) mecánicas de retroalimentación (en relación con el ciclo de retroalimentación en la mecánica de juego) y

c) mecánicas de progresión (acumulación de habilidades significativas).

Los componentes son las implementaciones específicas de las dinámicas y mecánicas: avatares, insignias, puntos, colecciones, rankings, niveles, equipos, bienes virtuales, etc. Hay unos componentes más po- 
pulares que otros, siendo los principales los puntos, las insignias y las tablas de clasificación ó PBLs (Points, achievement Badges \& Leader boards) (Werbach, 2012). Cabe destacar que los elementos no son el juego, lo que hace el juego es cómo estos elementos se entrelazan para conseguir que el jugador o jugadora se divierta.

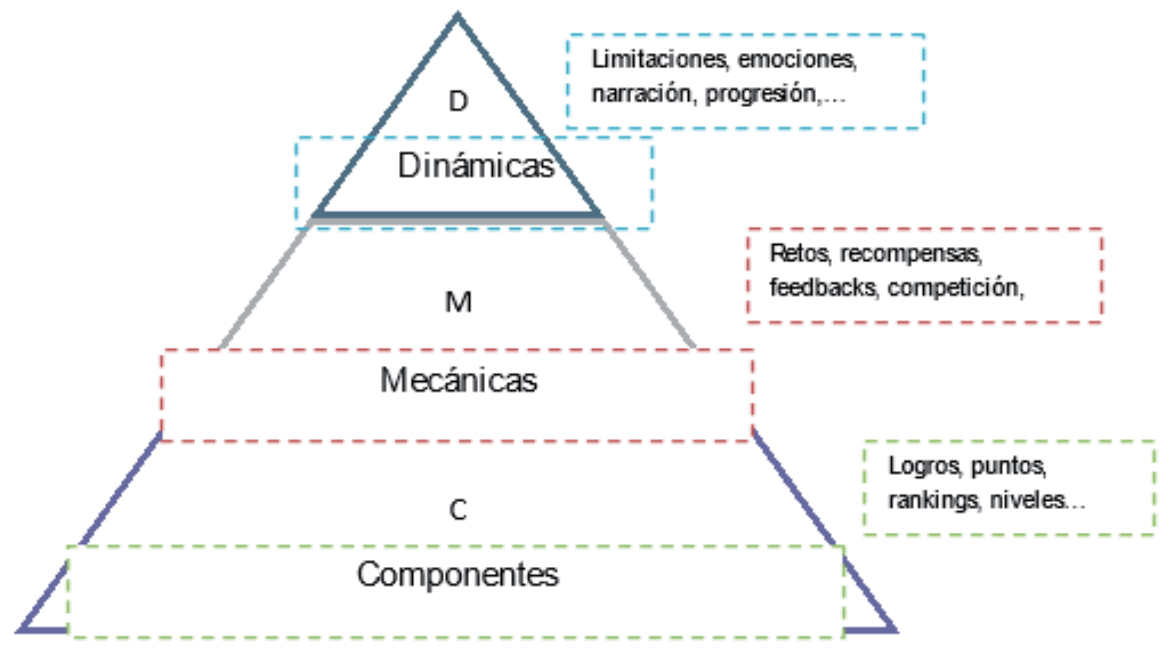

Figura 2. Pirámide de los Elementos de Gamificación. Adaptado de Kevin Werbach (2012).

En una revisión de la literatura sobre gamificación en la educación se ha encontrado que existen diversos elementos diseño para la gamificación de actividades educativas que poseen beneficios para el aprendizaje (Nah, Zeng, Telaprolu, Ayyappa \& Eschenbrenner, 2014). En la Tabla 1 se observan los elementos de diseño más utilizados en la gamificación de contextos educativos. 
Tabla 1. Resumen de elementos de diseño utilizados en trabajos relacionados de gamificación en educación (Adaptado de Nah et al., 2014).

\begin{tabular}{|c|c|c|}
\hline $\begin{array}{l}\text { Elementos de diseño para la } \\
\text { Gamificación }\end{array}$ & $\begin{array}{l}\text { Hallazgos -Beneficios } \\
\text { en el aprendizaje }\end{array}$ & $\begin{array}{l}\text { Referencia } \\
\text { Bibliográfica }\end{array}$ \\
\hline $\begin{array}{l}\text { - Puntos de experiencia } \\
\text { - Niveles } \\
\text { - Tablas de clasificación } \\
\text { - Desafíos } \\
\text { - Medallas }\end{array}$ & $\begin{array}{l}\text { Compromiso } \\
\text { Participación }\end{array}$ & Barata et al. (2013) \\
\hline $\begin{array}{l}\text { - Niveles } \\
\text { - Barras de progreso } \\
\text { - Puntos } \\
\text { - Feedback inmediato } \\
\text { - Tablas de clasificación } \\
\text { - Interacción de a pares y } \\
\text { colaboración } \\
\end{array}$ & & $\begin{array}{l}\text { Berkling \& Thomas } \\
\text { (2013) }\end{array}$ \\
\hline $\begin{array}{l}\text { - Puntos de experiencia } \\
\text { - Niveles } \\
\text { - Libre elección del nivel de } \\
\text { dificultad } \\
\end{array}$ & & Betts et al. (2013) \\
\hline $\begin{array}{l}\text { - Puntos } \\
\text { - Premios } \\
\end{array}$ & Motivación & Brewer et al. (2013) \\
\hline $\begin{array}{l}\text { - Puntos de experiencia } \\
\text { - Niveles } \\
\text { - Recompensas en el juego }\end{array}$ & $\begin{array}{l}\text { Compromiso } \\
\text { Diversión }\end{array}$ & $\begin{array}{l}\text { de Freitas } 6 \text { de Freitas } \\
\text { (2013) }\end{array}$ \\
\hline $\begin{array}{l}\text { - Integración (onboarding) } \\
\text { - Puntos } \\
\text { - Niveles } \\
\text { - Medallas } \\
\text { - Desafíos } \\
\text { - Repetir o hacer más } \\
\text { - Contenido desbloqueable } \\
\text { - Personalización }\end{array}$ & $\begin{array}{l}\text { Compromiso } \\
\text { Diversión } \\
\text { Experiencia de apren- } \\
\text { dizaje productiva }\end{array}$ & Eleftheria et al. (2013) \\
\hline - Medallas & $\begin{array}{l}\text { Motivación } \\
\text { Compromiso } \\
\text { Sentido de logro/com- } \\
\text { petencia } \\
\text { Estatus } \\
\end{array}$ & Gibson et al. (2013) \\
\hline $\begin{array}{l}\text { - Puntos de experiencia } \\
\text { - Niveles } \\
\text { - Logros }\end{array}$ & $\begin{array}{l}\text { Compromiso } \\
\text { Sentido de logro/com- } \\
\text { petencia }\end{array}$ & Goehle (2013) \\
\hline $\begin{array}{l}\text { - Narrativa (storytelling) } \\
\text { - Feedback }\end{array}$ & Compromiso & Kapp $(2012 a, b)$ \\
\hline
\end{tabular}




\begin{tabular}{|c|c|c|}
\hline $\begin{array}{l}\text { - Puntos } \\
\text { - Niveles } \\
\text { - Medallas } \\
\text { - Fases } \\
\end{array}$ & Compromiso & $\begin{array}{l}\text { Kumar \& Khurama } \\
(2012)\end{array}$ \\
\hline $\begin{array}{l}\text { - Historia (storyline) } \\
\text { - Elementos visuales } \\
\text { - Objetivos } \\
\text { - Recompensas } \\
\text { - Puntos } \\
\text { - Barras de progreso } \\
\text { - Medallas } \\
\text { - Tablas de clasificación }\end{array}$ & $\begin{array}{l}\text { Compromiso } \\
\text { Rendimiento }\end{array}$ & $\begin{array}{l}\text { O'Donovan et al. } \\
(2013)\end{array}$ \\
\hline $\begin{array}{l}\text { - Feedback frecuente } \\
\text { - Barras de progreso } \\
\text { - Recompensas } \\
\text { - Actualizaciones del per- } \\
\text { - } \text { sonaje } \\
\text { - Motivación de pares } \\
\end{array}$ & Compromiso & Raymer (2013) \\
\hline - Medallas & $\begin{array}{l}\text { Logro } \\
\text { Compromiso } \\
\text { Motivación } \\
\text { Reconocimiento } \\
\end{array}$ & Santos et al.(2013) \\
\hline $\begin{array}{l}\text { - Avatar } \\
\text { - Feedback } \\
\text { - Puntos } \\
\text { - Medallas } \\
\text { - Recompensas } \\
\end{array}$ & Interés en el curso & Todor \& Pitic (2013) \\
\hline $\begin{array}{l}\text { - Historia } \\
\text { - Tabla de puntuaciones }\end{array}$ & $\begin{array}{l}\text { Compromiso } \\
\text { Motivación }\end{array}$ & $\begin{array}{l}\text { Villagrasa \& Duran } \\
(2013)\end{array}$ \\
\hline
\end{tabular}

Sin embargo, también se han identificado algunos problemas en la introducción de la gamificación en los procesos de enseñanza aprendizaje, tales como: el exceso de carga de trabajo, tablas de comparaciones molestas o dolorosas, premios a la cantidad no a la calidad de las contribuciones o participación, despertar demasiado tarde y no poder recuperar el tiempo perdido en el juego (puntos de experiencia) y que se incentiva la competición frente a la colaboración y esto no permite que se premien a los estudiantes que se ayudan mutuamente (Barata et al, 2013). A partir de los problemas anteriores se proponen un conjunto de consideraciones de diseño que deben tenerse en cuenta a la hora gamificar actividades educativas, como por ejemplo las siguientes: 
a. Aligerar el ritmo y carga de trabajo. La carga de trabajo se debe manejar con cuidado. Los intervalos entre las tareas deben ser cuidadosamente elegidos para un mejor equilibrio con las facetas del juego.

b. Tener cuidado con las comparaciones. Considerar la posibilidad de diferentes tipos de clasificaciones, por ejemplo mostrar sólo los grupos más cercanos, crear diferentes "ligas", etc.

c. Calidad de la recompensa: Estimar la calidad de la participación y adjudicar los puntos de experiencia de cada estudiante en consecuencia. Esto aumentará la cantidad de trabajo realizado por los profesores, pero es un requisito para la equidad percibida del curso.

d. Hacerles participar tan pronto como sea posible. Muchos estudiantes sólo quieren empezar el juego cuando ya es demasiado tarde. Por ello, realizar una adaptación de la experiencia de juego de modo que se ven obligados a participar pronto (y ver las recompensas significativas) darán mejores resultados.

e. Darles la oportunidad de recuperar el tiempo perdido. Mientras que algunas tareas y desafíos siempre serán de una duración determinada, siempre que sea posible se debe permitir a los estudiantes hacer frente a los distintos retos de una manera restringida.

f. Proporcionar medios para la cooperación. Encontrar mecanismos donde varios estudiantes puedan trabajar juntos hacia un objetivo común, pero mantener la capacidad para que los estudiantes puedan mostrar su trabajo de forma individual.

g. Que la actividad y la evaluación sea sobre el juego. Varios estudiantes pueden pensar que pueden descuidar el juego, ya que pueden utilizar algunos componentes de evaluación tradicionales (tales como el examen).

Por otra parte, para gamificar una actividad es necesario encontrar la forma correcta de motivar a la persona adecuada en el momento adecuado y tener en cuenta que la motivación puede ser de dos tipos diferentes:

- Intrínsecas: inherente a la persona, lo realiza por su propio bien o interés (por ejemplo: estatus, poder, acceso a ciertas aptitudes) o para contribuir a un bien común

- Extrínsecas: exterior a la persona, lo realiza por la recompensa o feedback. 
También es importante tener en cuenta el componente social, o lo que es lo mismo, el contar con otras personas con las que competir, colaborar y comparar logros. En el juego social, los objetivos pueden ser competitivos o colaborativos. Por ello, en juegos de equipo deben ser consideradas por separado las mecánicas que influyen en el equipo (proyectos, puntuaciones de grupo, etc.), de las mecánicas que solo se aplican al individuo (motivación, el refuerzo positivo, etc.).

Por otra parte, es bueno seguir algunas pautas para la gamificación de actividades educativas, tales como las siguientes (Linehan et al., 2011):

- Experimentación repetida: se debe permitir que el estudiante-jugador pueda realizar repeticiones de la actividad para alcanzar una meta.

- Inclusión de ciclos de retroalimentación rápida: proporcionar información inmediata que ayude a los estudiantes a mejorar su estrategia y tener una mejor oportunidad de éxito en el siguiente intento.

- Adaptación de las tareas a los niveles de habilidad: los buenos juegos ayudan a los jugadores a estimar de manera realista sus posibilidades de éxito, los diferentes niveles con objetivos adaptados a las habilidades de los estudiantes permite mejorar su motivación.

- Intensificación progresiva de la dificultad de las tareas: ayuda a los estudiantes a mejorar sus habilidades y suponen nuevos retos.

- División de tareas complejas en subtareas más cortas y simples: esto ayuda a los estudiantes a hacer frente a la complejidad de la tarea.

- Diseño de diferentes rutas hacia el éxito: la planificación de diferentes formas de alcanzar los objetivos, como forma de personalización de las actividades.

- Incorporación de recompensas y actividades de reconocimiento social (por ejemplo, profesores y compañeros): ser recompensado y valorado promueve el estatus social de los estudiantes.

Creemos por tanto que muchas de las técnicas utilizadas en la gamificación de procesos pueden convertirse en estrategias útiles para ser aplicadas en las actividades de aprendizaje, tanto dentro del aula como fuera de ella, potenciando la motivación de los estudiantes hacia el aprendizaje. Además, es muy importante prestar especial atención a la narrativa en el diseño de los libros de texto, tan importante en el diseño de videojue- 
gos, ya que puede aumentar la motivación de los niños y niñas a partir de un elemento intrínseco: la curiosidad.

\section{Conclusiones}

Las reglas de juego de la cultura y el conocimiento, en este inicio del siglo XXI, han cambiado radicalmente. La digitalización de la información, la omnipresencia de las tecnologías en todos los ámbitos de vida social y personal, la conectividad permanente y acceso a la nube, la aparición de nuevos formatos y códigos de representación como el multimedia, la hipertextualidad, la realidad virtual o los escenarios tridimensionales, el incremento de la interactividad entre humano y máquina, la viralidad y réplica de cualquier información de modo masivo e instantáneo, así como el intercambio y flujo comunicativo entre las personas sin límites temporales y físicos ha provocado que los medios y formas culturales dominantes en la comunicación del siglo XX están en crisis. Las industrias culturales de la prensa, del cine y audiovisuales, de la música, de los libros y que fueron hegemónicas hasta hace pocos años han entrado en recesión. Por el contrario, industrias como la de los videojuegos o de las aplicaciones informáticas están en crecimiento y copando los mercados. De modo similar, aunque tardío, esta oleada de cambios está llegando e impactando sobre los medios y materiales educativos.

Las políticas de incorporación de las TIC a las escuelas impulsadas en numerosos países, sobre todo bajo el modelo 1:1, junto con el desarrollo de las competencias digitales tanto en el profesorado como el alumnado - los cuales son ya usuarios habituales de las tecnologías en su vida cotidiana- están propiciando que el monopolio de los libros de texto como principal material escolar empiece a menguar y ser cuestionado.

En el contexto internacional varios países ya han anunciado la implantación de políticas educativas destinadas a sustituir los libros de texto por materiales educativos digitales. Por ejemplo, en Corea del Sur el Ministerio de Educación han planificado que las tabletas y otros dispositivos electrónicos sustituirán a los libros de texto de papel en los próximos años. En USA varios estados federales como Florida o California ya han comenzado este proceso. Incluso la SETDA (Asociación de directores de tecnología educativa de los estados) reclaman que este proceso acabe en el curso 2017-2018. En el contexto español, las autoridades ministe- 
riales ya han empezado a reducir las subvenciones a la adquisición de los textos escolares a favor de lo que se denomina "mochila digital". En este sentido, las industrias editoras también han empezado a apostar por la publicación de lo que se denominan "contenidos educativos digitales" o "plataformas educativas".

Es indudable, en este siglo XXI, la necesidad y urgencia de que la escuela se apropie de la tecnología digital y transforme de modo radical su práctica pedagógica. Sin embargo, la mera sustitución de un tipo de tecnología por otra tecnología sabemos que no transforma las formas de enseñar ni los procesos de aprender. El cambio e innovación pedagógica requiere de más variables que el mero hecho de mudar de un tipo de soporte impreso a otro digitalizado. El cambio sustantivo está en la transformación de los formatos de representación y organización del conocimiento así como en la naturaleza de la interacción de los sujetos con dichas tecnologías.

En este artículo hemos visto que los libros de texto responden a una visión estructurada del conocimiento que favorecen un modelo enciclopedista y cerrado del conocimiento y que implica un aprendizaje por recepción de la información. Este tipo de material educativo responde a un modelo de tecnología impresa que además ha sido apropiado para la escolaridad del siglo XX. Sin embargo, con los cambios estructurales en la cultura y la tecnología del siglo XXI es imprescindible que los materiales educativos empiecen a adoptar, entre otros rasgos, una concepción gamificada del aprendizaje de modo que ofrezcan a los estudiantes oportunidades para el desarrollo de experiencias abiertas, flexibles, interactivas y lúdicas de aprendizaje de modo similar a las experiencias que tienen con los videojuegos. Las implicaciones que tienen este nuevo planteamiento gamificado son indudables. Por una parte, las industrias editoras deben experimentar con esta nueva lógica de productos educativos lo que implica por una parte, que participen nuevos profesionales que dominen tanto la ingeniería informática, la creación de interfaces digitales así como la construcción de narrativas potencialmente educativas. Por otra, estas industrias tienen que adoptar modelos de creación y desarrollo de los materiales distintos de los modelos tradicionales de producción en masa (del autor al editor y de éste al cliente), a adoptar modelos de investigación y desarrollo permanente de sus productos donde los usuarios son un elemento clave en el testeo y mejora de los materiales. 
Las implicaciones del enfoque de la gamificación para la docencia y el aprendizaje escolar son indudables. Supone que el modelo de enseñanza por exposición y del aprendizaje por recepción a través de materiales estandarizados, iguales para todos y altamente estructurados, como son los libros de texto, son profundamente cuestionados en su raíz. La naturaleza y la lógica de los materiales educativos gamificados, por el contrario, pone el acento en la experiencia interactiva del sujeto, en su implicación y toma de decisiones autónoma con relación al objeto de conocimiento. No lo recibe de forma homogénea y pasiva, sino que lo construye a través de la acción personalizada del juego en un entorno digitalizado.

Hemos visto además que existen diversos elementos que deben considerarse para la construcción de una actividad educativa gamificada, los cuales pueden tener efectos motivantes en la experiencia de aprendizaje de los estudiantes.

Somos conscientes de que todavía queda mucho camino por recorrer para que la gamificación de los materiales educativos deje de ser un experimento o una rareza en el actual panorama educativo y se conviertan en un fenómeno habitual de las escuelas. Pero es una tendencia que probablemente irá en crecimiento en los próximos años.

\section{Referencias bibliográficas}

Area, M. y Sanabria, A.L. (2014): Opiniones, expectativas y valoraciones del profesorado participante en el Programa Escuela 2.0 en España. Educar, vol. 50/1, 15-39. Recuperado de http://ddd.uab.cat/pub/educar/educar_a2014m1-6v50n1/educar_a2014m16v50n1p15.pdf 30/10/2014

Balanskat, A., Bannister, D., Hertz, B., Sigillò, E., \& Vuorikari, R. (2013). Overview and Analysis of 1:1 Learning Initiatives in Europe. JRC Scientific and Policy Report. Luxembourg: Publications Office of the European Union.

Barata, G., Gama, S., Jorge, J., Goncalves, D. (2013). Engaging Engineering Students with Gamification. 5th International Conference on Games and Virtual Worlds for Serious Applications (VS-GAMES), IEEE, pgs. 1-8

Berkling, K., Thomas, C. (2013). Gamification of a Software Engineering Course. In: International Conference on Interactive Collaborative Learning, pp. 525-530.

Betts, B.W., Bal, J., Betts, A.W. (2013). Gamification as a Tool for Increasing the Depth of Student Understanding using a Collaborative E-learning Environment. International Journal of Continuing Engineering Education and Life-Long Learning 23(3-4), 213-228.

Brewer, R., Anthony, L., Brown, Q., Irwin, G., Nias, J., Tate, B. (2013). Using Gamifica- 
De la enseñanza con libros de texto al aprendizaje en espacios online gamificados

tion to Motivate Children to Complete Empirical Studies in Lab Environments. In: 12th International Conference on Interaction Design and Children, pp. 388-391.

Cook, W. (2013) Training Today: 5 Gamification Pitfalls. Training Magazine. Recuperado de http://www.trainingmag.com/content/training-today-5-gamification-pitfall. $15 / 10 / 2014$

De Freitas, A.A., de Freitas, M.M. (2013). Classroom Live: A Software-assisted Gamification Tool. Computer Science Education 23(2), 186-206.

Eleftheria, C.A., Charikleia, P., Iason, C.G., Athanasios, T., Dimitrios, T. (2013). An Innovative Augmented Reality Educational Platform using Gamification to Enhance Lifelong Learning and Cultural Education. In: 4th International Conference on Information, Intelligence, Systems and Applications, pp. 1-5.

Fitz-Walter Z. \& Tjondronegoro D, Wyeth P. (2011). Orientation Passport: using gamification to engage university students. Proceedings of the 23rd Australian ComputerHuman Interaction Conference, ACM, pgs. 122-125.

Fletcher, G.; Schaffhauser, D. y Levin, D. (2012): Out of print: Reimagining the K-12 textbook in digital age. SETDA, Stte Educational Technology Directors Association, Washington, D.C.

Gartner R. (2014). Hype Cycle for Emerging Technologies. Recuperado de http://www. gartner.com/technology/research/hype-cycles/ 5/11/2014

Gee, J. P. (2003). What Digital Games Have to Teach Us. About Learning and Literacy. New York \& Basingstoke: Palgrave Macmillan

Gibson, D., Ostashewski, N., Flintoff, K., Grant, S., Knight, E. (2013). Digital Badges in Education. Education and Information Technology. Springer, New York.

Gimeno (1988): El curriculum: una reflexión sobre la práctica. Morata, Madrid.

Goehle, G. (2013). Gamification and Web-based Homework. Problems, Resources, and Issues in Mathematics Undergraduate Studies 23(3), 234-246.

González C. S. \& Mora A. (2014). Methodological proposal for gamification in the computer engineering teaching. En Proceeding Computers in Education (SIIE), 2014 International Symposium on. Pages 29-34. IEEE C2014

González C.S. \& Blanco F. (2008). Integrating an educational 3D game in Moodle. Simulation \& Gaming. SAGE Publications.

González C.S. \& Blanco F. (2012). Designing social videogames for educational uses. Computers \& Education. Volúmen 58. Número 1. Páginas 250-262.

Kapp, K.M. (2012a).: Games, Gamification, and the Quest for Learner Engagement. Training and Development 66(6), 64-68.

Kapp, Karl. (2012b) The Gamification of Learning and Instruction: Game-Based Methods and Strategies for Training and Education. San Francisco: Pfeiffer.

Klassen, D. y Solid, M. (1981): Toward an Appropiate Technology for Education. Educational Technology. oct. pp. $28-31$

Kumar, B., Khurana, P. (2012). Gamification in Education - Learn Computer Programming with Fun. International Journal of Computers and Distributed Systems 2(1), 46-53.

Lee, J. \& Hammer, J. (2011). Gamification in Education: What, How, Why Bother? Academic Exchange Quarterly, 15(2)

Linehan C., Kirman B., Lawson S., Chan G. (2011). Practical, appropriate, empirically- 
De la enseñanza con libros de texto al aprendizaje en espacios online gamificados Manuel Area Moreira y Carina S. González González

validated guidelines for designing educational games. Proceedings of the SIGCHI Conference on Human Factors in Computing Systems.

Martínez Bonafé, J. (2001): Óxido sobre el curriculum en plena era digital (Crítica a la tecnología del libro de texto). Kikiriki. Cooperación educativa, № 61, 2001, págs. 22-29

Martínez Bonafé, J. (2008) Los libros de texto como práctica discursiva. Revista de la Asociación de Sociología de la Educación. Vol. 1, №. 1, 2008, págs. 62-73. Recuperado de http://dialnet.unirioja.es/descarga/articulo/2793153.pdf 23/09/2012

Martínez Bonafé, J. (2010): El currículum y el libro de texto. una dialéctica siempre abierta. En J. Gimeno (Coor): Saberes e incertidumbres sobre el currículum. Morata, Madrid.

McFarlane, A., Sparrowhawk, A.\& Heald, Y. (2002). Report on the educational use of games: An exploration by TEEM of the contribution which games can make to the education process. Recuperado de http://www.kennisnet.nl/uploads/tx_kncontentelements/games_in_education_full1.pdf 8/1/2014

Mérida, R., Angulo, J., Jurado, M., Diaz, J. (2011). Student Training in Transversal Competences at the University of Cordoba. European Educational Research Journal, 10 (1), 34-52.

Merquis J. (2013). 5 Easy Steps to Gamifying Higher Education. Recuperado de http:// classroom-aid.com/2013/08/16/5-easy-steps-to-gamifying-highereed/ 3/11/2014

Nah F., Zeng Q., Telaprolu V., Ayyappa A. , Eschenbrenner B. (2014). Gamification of Education: A Review of Literature. $\mathrm{HCl}$ in Business. First International Conference, HCIB 2014. Held as Part of HCI International 2014 Heraklion, Crete, Greece, June 22-27, 2014 Proceedings. Pgs. 401-409.

O'Donovan, S., Gain, J., Marais, P. (2013). A Case Study in the Gamification of a University level Games Development Course. In: Proceedings of the South African Institute for Computer Scientists and Information Technologists Conference, pp. 242-251.

OCDE (2001): Schooling for Tomorrow. What Schools for the Future?. Centre for Educational Research and Innovation (CERI), Paris.

OCDE (2009): Beyond Textbooks: Digital Learning Resources as Systemic Innovation in the Nordic Countries. Centre for Educational Research and Innovation, Paris. Recuperado de http://www.keepeek.com/Digital-Asset-Management/oecd/education/ beyond-textbooks_9789264067813-en 3/10/2012.

Raymer, R. (2011). Gamification - Using Game Mechanics to Enhance eLearning. eLearn Magazine. Recuperado de http://elearnmag.acm.org/featured.cfm?aid=2031772 13/09/2013

Santos, C., Almeida, S., Pedro, L., Aresta, M., Koch-Grunberg, T. (2013). Students' Perspectives on Badges in Educational Social Media Platforms: The Case of SAPO Campus Tutorial Badges. In: IEEE 13th International Conference on Advanced Learning Technologies, pp. 351-353.

Todor, V., Pitic , D. (2013). The Gamification of the Study of Electronics in Dedicated Elearning Platforms. In: 36th International Spring Seminar on Electronics Technology, pp. 428-431.

Villagrasa, S., Duran, J. (2013). Gamification for Learning 3D Computer Graphics Arts. In: 
De la enseñanza con libros de texto al aprendizaje en espacios online gamificados

Manuel Area Moreira y Carina S. González González

First International Conference on Technological Ecosystem for Enhancing Multiculturality, pp. 429-433.

Werbach, K. (2012) For the Win: How Game Thinking Can Revolutionize Your Business. Wharton: Wharton Digital Press, 2012.

Zhijiang Dong C.L., Untch R., \& Chasteen M. (2013). Engaging Computer Science Students through Gamification in an Online Social Network Based Collaborative Learning Environment. International Journal of Information and Education Technology, vol. 3, no. 1, pp. 72-77, 2013. 
